DOI: 10.12731/wsd-2018-2-108-115

УДК 616.839-02:616.12-008.331.1-03

\title{
ФУНКЦИОНАЛЬНОЕ СОСТОЯНИЕ ВЕГЕТАТИВНОЙ НЕРВНОЙ СИСТЕМЫ У КРЫС, ПОСЛЕ ВДЫХАНИЯ ВЫСОКОДИСПЕРСНОГО АЭРОЗОЛЯ ИЗ ЭЛЕКТРОННОЙ СИСТЕМЫ ДОСТАВКИ НИКОТИНА
}

Королев И.Б., Подкаура О.В.

Целью исследования послужило изучение функционального состояния вегетативной нервной системы у крыс, после вдыхания высокодисперсного аэрозоля из электронной системы доставки никотина. Исследование проведено на 20 половозрелых особях. Выявлена инверсия чувствительности вегетативной нервной системы у крыс при действии высокодисперсного аэрозоля, поступающего из прибора, имитирующего электронную сигарету, при однократном воздействии регистрировался функииональный дисбаланс вегетативной регуляции ритма сердиа с преобладанием парасимпатикотонии и недостаточностью нейрогуморальной авторегуляиии миокарда на фоне ичентрализации управления работой сердияа при выраженной флуктуачии адренергического компонента. С увеличением экспозиции при многократных ингаляциях наблюдалась выраженная дезадаптация животных, что ассоииируется с дисфункиией вегетативной нервной системы в виде снижениения активности парасимпатического отдела и повышения тонуса симпатического отдела.

Ключевые слова: крыса; вейп; вейпинг; вегетативная нервная система; вариабельность ритма сердиа.

\section{FUNCTIONAL STATE OF THE AUTONOMIC NERVOUS SYSTEM IN RATS AFTER INHALING A HIGHLY DISPERSED AEROSOL FROM THE ELECTRONIC NICOTINE DELIVERY SYSTEM}

Korolev I.B., Podkaura O.V.

The purpose of the study was to study the functional state of the autonomic nervous system in rats after inhaling a highly dispersed aerosol from the elec- 
tronic nicotine delivery system. The study was conducted on 20 mature rats. The inversion of the sensitivity of the vegetative nervous system in rats is revealed when a highly dispersed aerosol (vapor) comes from the device simulating an electronic cigarette. With a single exposure to aerosol (steam), a functional imbalance in vegetative regulation of the heart rhythm with a predominance of parasympathicotonia and a failure of neurohumoral autoregulation of the myocardium was recorded in the animals against the backdrop of centralized control of cardiac function against the background of pronounced fluctuations in the adrenergic component. With the increase in exposure to multiple inhalations of aerosol (vapor), pronounced maladaptation of animals is observed, which is associated with autonomic nervous system dysfunction in the form of a decrease in parasympathetic activity and an increase in the tone of the sympathetic department.

Keywords: rat; wap; wapping; autonomic nervous system; heart rate variability.

Для многих молодых людей весьма заманчива возможность парить без ограничений, но есть опасения, что высокодисперсный аэрозоль, содержащийся в электронных сигаретах и используемый для генерации пара, может вызвать нарушение деятельности вегетативной нервной системы и сердечного ритма [5].

Анализ вариабельности сердечного ритма (ВСР) является одним из наиболее информационных методов оценки состояния симпатического и парасимпатического тонуса, который позволяет судить о состоянии механизмов регуляции физиологических функций в организме человека, вести мониторинг текущего гомеостаза организма и его адаптационных резервов, дать прогноз не только при возникновении заболевания, но и в донозологическом периоде [2].

В связи с вышеизложенным, было проведено исследование, направленное на изучение состояния вегетативной нервной системы у крыс, находящихся под воздействием высокодисперсного аэрозоля, содержащегося в электронных сигаретах, используемого для генерации пара.

\section{Материалы и методы исследования}

В эксперименте использовано 20 половозрелых (3-3,5 мес.) самцов

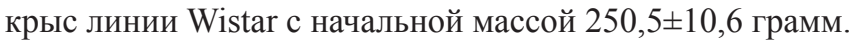

На первом этапе исследования проанализировано 53 электрокардиограммы. Определены параметры временных и спектральных показателей ритма сердца, у 20 крыс. 
На втором этапе животные были разделены на 4 группы, в контрольную группу вошли 5 крыс, в опытную - 15. Опытная группа была поделена на 3 подгруппы по 5 особей. На данном этапе были созданы условия поступления высокодисперсного аэрозоля в дыхательные пути крыс, из прибора, имитирующего электронную сигарету.

Первая подгруппа животных находилась под воздействием пара 10 минут, вторая - 20, третья - 30 минут. Длительность эксперимента составила 30 суток, проанализировано 446 электрокардиограмм.

Измерение ВРС выполняли при помощи ветеринарного электрокардиографа Поли-Спектр-8/В (НейроСофт, Россия).

Вегетативную регуляцию сердца оценивали по стандартам Европейского кардиологического и Североамериканского электрофизиологического обществ (2007).

Спектральный анализ вариабельности ряда RR-интервалов у крыс проводили на основе алгоритма быстрого преобразования Фурье с использованием всех точек без сглаживания. Оценивали следующие показатели: общую мощность спектра колебаний сердечного ритма (ТР, 0,003-2,5 Гц); мощность высокочастотной составляющей (HF, 0,78-2,5 Гц); мощность в диапазоне низких частот (LF, 0,195-0,74 Гц) и их нормированные значения (LF, n.u. и HF, n.u.); мощность в диапазоне очень низких частот (VLF, 0,003-0,195 Гц). Кроме того, определяли симпатико-парасимпатическое взаимодействие (LF/HF), характеризующееся отношением низких (LF) и высоких частот (HF) спектра.

Данные, полученные в исследовании, обобщались и представлялись путем вычисления среднего значения и его ошибки $(\mathrm{M} \pm \mathrm{m})$. Определение нормальности выборок проводили путем проведения теста Шапиро-Уилкса. Распределение считали нормальным при $p>0,05$. Достоверность разницы показателей, полученных в группах сравнения, определяли с помощью $t$-теста Стьюдента для выборок с нормальным распределением и теста Крускала-Уоллиса, U-теста Манна-Уитни для выборок с распределением, отличавшимся от нормального. Корреляционный анализ проводился путем вычисления коэффициента корреляции Спирмена. В исследовании был принят критический уровень достоверности 0,05 .

\section{Результаты исследования и их обсуждение}

В процессе изучения показателей временного и спектрального анализа сердечного ритма были выявлены изменения в системе вегетативной регуляции сердечного ритма у опытной группы крыс. К таким особенностям 
относится чувствительность показателей ССС как индикатора адаптационных реакций всего организма.

У животных опытных групп после экспозиции паром показатели временного ряда отражали снижение механизмов вегетативной регуляции функции кровообращения по сравнению с показателями, зарегистрированными в контрольной группе.

Частота сердечных сокращений в опытной группе крыс №1 в показателях абсолютного прироста достоверно увеличилась на 8,0\%. По данным временного анализа вдыхание пара в течении 10 минут меньше всего оказало влияние на общее состояние гуморального канала регуляции, при этом средняя длительность средних интервалов RR (RRNN) достоверно уменьшилась на 18,5\% (табл. 1), что указывает на вегетативную дисфункцию, за счет усиления симпатического влияния и включения в регуляцию надсегментарных уровней управления ритмом сердца на фоне подавления его автономного контура управления.

Уменьшение вариабельности длины волны в стандартном отклонении всех NN интервалов $(\mathrm{SDNN})$ на 7,6\% (p < 0,001) и на 7,3\% (p < 0,05) квадратного корня из суммы квадратов разностей величин последовательных пар RR-интервалов (RMSSD) свидетельствует об угнетении общего тонуса парасимпатического отдела вегетативной нервной системы. После вдыхания аэрозоля у крыс опытной группы №1 прослеживается снижение адаптации сердечно-сосудистой системы в виде возможности колебания средних величин ряда по коэффициенту вариабельности (CV, \%) на 8,5\% $(\mathrm{p}<0,01)$ по сравнению с исходными величинами. Уменьшение показателя pNN5 \% - процент от общего количества последовательных пар интервалов, различающихся более чем на 5 мс, полученных за весь период записи - в 3 раза $(\mathrm{p}<0,01)$ подтверждает блокаду парасимпатического отдела вегетативной нервной системы под действием препарата.

При анализе частотных составлявших спектра наблюдается незначительное уменьшение низкочастотной (LF) составляющей спектра свидетельствующее об ослаблении симпатической активности и усилении парасимпатической регуляции и общей мощности колебаний кардиоритма (TP) на 3,3\%. Соотношение низких и высоких частот LF/HF на 21,2\% $(\mathrm{p}<0,001)$, указывает на преобладание в спектре низкочастотного компонента. Мощность спектров сверхнизких (VLF) и высоких частот (HF) снизилась в 2,16 раза $(\mathrm{p}<0,05)$ и на $6,5 \%(\mathrm{p}<0,01)$ соответственно. Снижение спектра очень низких частот свидетельствует о существенном постнагрузочном энергетическом дефиците. 
Таблийа 1.

Показатели вариабельности сердечного ритма крыс в зависимости от времени экспозиции паром (аэрозолем) от электронных систем подачи никотина $(\mathrm{M} \pm \mathbf{m})$

\begin{tabular}{|c|c|c|c|c|}
\hline \multirow[b]{2}{*}{ показатель } & \multirow[b]{2}{*}{$\begin{array}{c}\text { контрольная } \\
\text { группа }\end{array}$} & \multicolumn{3}{|c|}{ Опытная группа / время экспозиции } \\
\hline & & $\begin{array}{l}\text { опытная груп- } \\
\text { па №1 (экспо- } \\
\text { зиция } 10 \text { мин) }\end{array}$ & $\begin{array}{l}\text { опытная груп- } \\
\text { па №2 (экспо- } \\
\text { зиция } 20 \text { мин) }\end{array}$ & $\begin{array}{c}\text { опытная группа } \\
\text { № } 3 \text { (экспози- } \\
\text { ция } 30 \text { мин) }\end{array}$ \\
\hline $\begin{array}{c}\text { ЧСС, уд/ } \\
\text { мин }\end{array}$ & $395,28 \pm 8,95$ & $429,46 \pm 17,17 * *$ & $382,53 \pm 12,88 * *$ & $345,94 \pm 17,37 *$ \\
\hline RRNN, Mc & $152,77 \pm 12,01$ & $124,09 \pm 11,42 * *$ & $140,17 \pm 12,60 *$ & $157,78 \pm 12,23 *$ \\
\hline SDNN, Mc & $5,65 \pm 0,30$ & $5,22 \pm 0,79 *$ & $12,78 \pm 1,28 * * *$ & $33,49 \pm 5,01 *$ \\
\hline $\begin{array}{l}\text { RMSSD, } \\
\text { мс }\end{array}$ & $8,81 \pm 0,39$ & $8,17 \pm 0,41 * * *$ & $16,11 \pm 1,49 * *$ & $49,66 \pm 6,38 * * *$ \\
\hline $\mathrm{pNN} 5, \%$ & $0,39 \pm 0,16$ & $1,18 \pm 0,49 *$ & $3,45 \pm 0,57 *$ & $4,58 \pm 0,73^{*}$ \\
\hline $\mathrm{CV}, \%$ & $3,02 \pm 0,12$ & $3,30 \pm 11,36 * * *$ & $6,67 \pm 0,56^{* *}$ & $20,76 \pm 2,22 * * *$ \\
\hline $\mathrm{TP}, \mathrm{Mc}^{2}$ & $240,51 \pm 10,16$ & $232,50 \pm 13,34 * *$ & $241,03 \pm 2,68 * *$ & $281,12 \pm 12,64^{*}$ \\
\hline VLF, $\mathrm{Mc}^{2}$ & $7,06 \pm 1,75$ & $3,26 \pm 0,22 *$ & $5,52 \pm 0,94 * *$ & $10,34 \pm 0,22 *$ \\
\hline $\mathrm{LF}, \mathrm{Mc}^{2}$ & $114,89 \pm 12,15$ & $112,80 \pm 8,03 * *$ & $156,88 \pm 10,78 * *$ & $144,98 \pm 15,17 * * *$ \\
\hline $\mathrm{HF}, \mathrm{мc}^{2}$ & $126,07 \pm 13,16$ & $117,89 \pm 11,36^{*}$ & $162,10 \pm 13,89 * *$ & $197,91 \pm 17,14 * *$ \\
\hline $\mathrm{LF} / \mathrm{HF}$ & $0,52 \pm 0,04$ & $0,41 \pm 0,02 * *$ & $0,69 \pm 0,07 * *$ & $1,20 \pm 0,16^{* *}$ \\
\hline
\end{tabular}

Примечание: в этой и последующих таблицах достоверность различий * $-\mathrm{p}<0,05$; $* *-\mathrm{p}<0,01 ; * * *-\mathrm{p}<0,001 ; \mathrm{RRNN}-$ средняя длительность средних интервалов $\mathrm{RR}$; CV - коэффициент вариабельности; SDNN - стандартное отклонение всех NN интервалов; RMSSD - квадратный корень из суммы квадратов разностей величин последовательных пар RR-интервалов; pNN5 \% от общего количества последовательных пар интервалов, различающихся более чем на 5 мс, полученных за весь период записи; ТР - общая мощность спектра колебаний сердечного ритма; HF - мощность высокочастотной составляющей спектра; LF - мощность в диапазоне низких частот; VLF - мощность в диапазоне очень низких частот; LF/HF отношение спектральной мощности сердечного ритма в диапазоне низких LF и высоких НF частот.

Таким образом, у крыс опытной группы №1 при вдыхании пара (аэрозоля) от электронной система доставки никотина были выявлены существенные изменения показателей пульсометрии, подтверждающие несостоятельность нейрогуморальной авторегуляции кровообращения и усиление централизации управления работы сердца. Полученные данные подтверждают, что согласование регуляторных процессов автономного контура вегетативной регуляции сердца у крыс встречается с известными 
затруднениями, а это мобилизует надсегментарный уровень вегетативной нервной системы.

С увеличением времени воздействия аэрозоля до 20 и 30 минут регистрировались показатели имеющие существенные отклонения от показателей, зафиксированных в контрольной группе. При этом отмечалась постепенная стабилизация некоторых данных пульсометрии.

У опытной группы крыс № 3 после 30 минут экспозиции паром, уровень чистоты сердечных сокращений отличался от контрольной группы в сторону уменьшения на $12,5 \%$. По данным временного анализа увеличение RRNN в 1,3 раза, свидетельствует о преобладании гуморального канала регуляции. Повышение средней длительности средних интервалов RR $(\mathrm{RRNN})$, стандартного отклонения (SDNN) в 5,9 раза, суммарной эффективности вегетативного регулирования квадратного корня из суммы квадратов разностей величин последовательных пар RR-интервалов (RMSSD) в 5,6 раза и коэффициента вариабельности (CV) в 16,8 раза говорит о повышении активности парасимпатического звена вегетативной нервной системы. Увеличение в 6,3 раза процента (pNN5 \%) от общего количества последовательных пар интервалов доказывает выраженное угнетение симпатического отдела вегетативной нервной системы.

Спектр очень низких (VLF) и низких частот (LF) увеличился на $31,7 \%$ и $20,7 \%$ соответственно. Достоверное увеличение VLF и LF спектра колебаний сердечного ритма демонстрирует высокую лабильность вегетативной нервной системы по автономному контуру управления.

Таким образом показатели вариабельности сердечного ритма у крыс позволяют достоверно вести мониторинг функционального состояния миокарда и его адаптационного резерва. С увеличением времени воздействия аэрозоля обнаружено увеличение общей мощности ритма сердца и выраженная динамика вариабельности сердечного ритма, связанная с чрезмерной активностью парасимпатического отдела вегетативной нервной системы. Регистрируемые показатели выходят за границы нормативного диапазона, указывая на дезадаптацию животных в условиях ингаляции аэрозоля. Данные нашего эксперимента регистрируют усиление процессов регуляции и централизации управления ритмом сердца на фоне повышения напряжения регуляторных систем.

\section{Список литературы}

1. Бороноев В.В. Спектральный анализ вариабельности сердечного ритма по пульсовой волне при нагрузочных пробах / Бороноев В.В., Омпоков В.Д., 
Павлов А.Е. // Вестник Бурятского Медицинского Университета. Улан-Удэ. 2012. C. 223-226.

2. Королев И.Б., Котельников В.Н., Осипов И.О. Физиологическая оценка вегетативной регуляции сердца крыс // В мире научных открытий. Красноярск. 2012. № 2.3 (26). С. 43-56.

3. Кузнецов А.А. Связь между временными и структурно-топологическими характеристиками диаграмм ритма сердца здоровых людей // Информатика и ее применения. 2010. Т. 4. Вып. 4. С. 39-48.

4. Курьянова Е.В. Влияние наркоза и рефлекторной стимуляции симпатоадреналовой и парасимпатической систем на вариабельность сердечного ритма крыс // Естественные науки. 2011. № 02. С. 140-148.

5. Мащенко Д.В. Изменение поведенческих реакций у мышей на фоне воздействия традиционных и электронных сигарет / Мащенко Д.В., Коноваленков С.Н. // XVI межрегиональная научно-практическая конференция студентов и молодых ученых «Медицина завтрашнего дня». Чита. 2017. С. 291-292.

6. Стригина М.И. Исследование погрешности данных фотоплетизмограммы для анализа вариабельности сердечного ритма / Стригина М.И., Чайванов Д.Б., Чудина Ю.А.// Биомедицина. Москва. 2013. № 4. С. 139-148.

7. Чернозуб А.А. Вариабельность сердечного ритма у нетренированных юношей в условиях различных режимов силовой нагрузки // Вестник РАМН. 2014 г. № 1-2. C. 51-56.

8. Mohamad Sleiman. Emissions from Electronic Cigarettes: Key Parameters Affecting the Release of Harmful Chemicals / Mohamad Sleiman, Jennifer M. Logue, Nahuel Montesinos, Marion L. Russell, Marta I. Litter, Lara A. Gundel and Hugo Destaillats // Environmental Science \& Technology. 2016, pp. 1-8.

\section{References}

1. Boronoev V.V., Ompokov V.D., Pavlov A.E. Vestnik Buryatskogo Meditsinskogo Universiteta. Ulan-Ude. 2012, pp. 223-226.

2. Korolev I.B., Kotel'nikov V.N., Osipov I.O. V mire nauchnykh otkrytiy. Krasnoyarsk. 2012. № 2.3 (26), pp. 43-56.

3. Kuznetsov A.A. Informatika i ee primeneniya. 2010. V. 4. Issue 4, pp. 39-48.

4. Kur'yanova E.V. Estestvennye nauki. 2011. № 02, pp. 140-148.

5. Mashchenko D.V., Konovalenkov S.N. XVI mezhregional'naya nauchno-prakticheskaya konferentsiya studentov i molodykh uchenykh «Meditsina zavtrashnego dnya» [XVI interregional scientific and practical conference of students and young scientists "Medicine of Tomorrow"]. Chita. 2017, pp. 291-292.

6. Strigina M.I., Chayvanov D.B., Chudina Yu.A. Biomeditsina. Moscow. 2013. № 4, pp. 139-148. 
7. Chernozub A.A. Vestnik RAMN. 2014. № 1-2, pp. 51-56.

8. Mohamad Sleiman. Emissions from Electronic Cigarettes: Key Parameters Affecting the Release of Harmful Chemicals / Mohamad Sleiman, Jennifer M. Logue, Nahuel Montesinos, Marion L. Russell, Marta I. Litter, Lara A. Gundel and Hugo Destaillats. Environmental Science \& Technology. 2016, pp. 1-8.

\section{ДАННЫЕ ОБ АВТОРАХ}

Королев Игорь Борисович, начальник Учебного Военного Центра при ВГМУ, полковник м/с

ГБОУ ВПО Тихоокеанский государственный медицинский университет пр. Острякова, 2, г. Владивосток, Приморский край, 690002, Российская Федераиия fvokorolev@yandex.ru

Подкаура Оксана Владимировна, к.м.н., начальник медицинского пункта-врач-педиатр

Филиал федерального государственного казенного общеобразовательного учреждения «Нахимовское военно-морское училище Министерства обороны Российской Федерации» (Владивостокское президентское кадетское училище), г. Владивосток

Камский переулок, 6, г. Владивосток, Приморский край, 690062, Российская Федераиия oksana.vp282@yandex.ru

\section{DATA ABOUT THE AUTHORS}

Korolev Igor Borisovich Ph.D., Head of the Military Center Federal State Budget Educational Institution of Higher Education Pacific National Medical University of the Ministry of Health of the Russian Federation

2, Ostryakova Ave., Vladivostok, Primorsky Krai, 690002, Russian Federation fvokorolev@yandex.ru

Podkaura Oksana Vladimirovna, Ph.D., Head of the Medical unit-pediatrician Nakhimov Naval College of the Ministry of Defense of the Russian Federation (Vladivostok presidential cadet school)

6, Kamsky lane, Vladivostok, Primorsky Krai, 690062, Russian Federation oksana.vp282@yandex.ru 